\title{
Recent Advances in Transgenic Rice Research Technology
}

\author{
Nayan Tara ${ }^{1}$, Meena $^{2 *}$ and Manjjri Singal ${ }^{1}$ \\ ${ }^{1}$ Department of Biotechnology, CRM Jat College, Hisar-125004, Haryana, India \\ ${ }^{2}$ Microbial Resource Technology Laboratory, Dept. of Microbiology, Kurukshetra University \\ Kurukshetra-136119, India \\ *Corresponding author
}

\section{A B S T R A C T}

\section{Keywords}

Oryza sativa,

Transgenic rice,

Biotic stress

Article Info

Accepted:

28 February 2018

Available Online:

10 March 2018
Rice is an important staple food crops for more than half of the world's population. Rice (Oryza sativa) productivity is adversely impacted by numerous biotic and abiotic factors. An approximate $50 \%$ of the global rice production is hampered annually due to the damage caused by biotic factors, out of which $\sim 20 \%$ is due to the attack of insect pests. Insect pests are a major biotic constraint on rice production. Transgenic approach has been explored to confer insect pest resistance to rice plants. In this paper, we review the progress that has been made in last years insect resistant transgenic rice research.

\section{Introduction}

Rice (Oryza sativa L.) is one of the most important sources of calories to human population providing $23 \%$ calories globally (Khush, 2003). It is the third largest produced cereal crop after wheat and maize. It is estimated that by 2050 the world would be requiring $60 \%$ more food, feed and biomass from the same amount of the land (FAO Report, 2009). Among cereals and monocots, rice being one of the major food crop of the world, having relatively small genome size (389 Mb, International Rice Genome Sequencing Project, 2005), complete genome sequenced, with well-developed markers emerged as a model monocot system for studying gene expression and function and for genetic transformation (Tyagi et al., 1999; Giri and Laxmi, 2000; Bajaj and Mohanty, 2005). Green revolution has increased the global rice production from 257 million tons to 718 million tons since 1996 to 2011 (Wani and Sah, 2014). But still the food grain production is lagging behind due to increased demand due to several factors including continuous increase in global population, urbanization. The condition is further deteriorated by continuously decreasing arable land for crops, abiotic and biotic stress amongst many other factors. The two major biotic stresses, which greatly constrain rice production, are insect pests and weeds. In this regard plant tissue culture and transgenic 
approaches are powerful means to address these challenges by transferring target genes to host organisms through different strategies. The objective of this review is to trace the advancements in the field of transgenic insect resistant rice.

\section{Transgenic rice for insect resistance}

Insects not only damage the rice plant physiologically, but also act as a vector for various viral diseases (Kathuria et al., 2007). The leaf folders, plant hoppers, stem borers and gall midges are most common insect pest species (Maclean et al., 2002). Insect pest attack can cause rice yield loss up to $10 \%$ every year (Chen et al., 2009). As chemical insecticides pose risk to both environmental as well as human health, thus genetic engineering provide a safe way to develop insect resistance in rice by transferring genes from different sources. The genes from Bacillus thuringiensis have been used extensively in this regard. Majority of the Bacillus thuringiensis's cry proteins are harmful to Lepidopteran insect pests; however, some of them are also lethal to Coleopteran insect pests from (McPherson et al., 1988) or Diptera (Yamamoto and Mclaughlin, 1981). It has been reported that Bt proteins do not pose any risk to beneficial insects, other animals, or humans (Klausner, 1984).

There are different reports, in which Bacillus thuringiensis (Bt)-derived genes namely Cry genes have been introduced in rice against insect resistance via different methods of gene delivery electroporation (Fujimoto., 1993), biolistic method (Wunn et al., 1996; Nayak et al., 1997; Ghareyazie 1997; Alam et al., 1998; Datta et al., 1998; Alam et al., 1999); and by Agrobacterium method (Cheng et al., 1998). Fujimoto et al., (1993) were the first to successfully generate the insect-resistant transgenic rice by transferring modified crylAb gene from Bacillus thuringiensis.
Subsequently, several rice varieties were successfully transformed using variants of cry gene as shown in Table 1. In India, first transgenic rice expressing cry gene viz IR62, was produced Nayak et al., (1997). Cheng et al., (1998) produced transgenic rice plants via Agrobacterium-mediated transformation using two $B t$ gene synthetic $\operatorname{crylA}(b)$ and $\operatorname{crylA}(c)$. Maqbool et al., (1998) introduced novel endotoxin cry $2 A$ gene in Basmati 370 and M7 cultivars via particle bombardment method. Shu et al., (2000) successfully obtained highly pest resistance using synthetic $c r y l A b$ that showed resistance toeight lepidopteran pests, both in laboratory and natural conditions. However transgenic plants showed some variation in agronomic traits such as seedling growth and yield (Shu et al., 2002). Chen et al., (2005) produced transgenic Indica rice with enhanced resistance against lepidopteron pests using synthetic cry $2 A$ of Bacillus thuringiensis. Tang et al., 2006 developed insect-resistant transgenic indica rice variety namely, Minghui 63 with a synthetic crylC gene. The resistant gene, crylC, driven by the rice $\mathrm{rbcS}$ promoter (small subunit of ribulose1,5-bisphosphate carboxylase/oxygenase), was introduced into Zhonghua 11 (Oryza sativa L. ssp. Japonica) via Agrobacterium-mediated transformation (Ye et al., 2009). Yarasi et al., (2008), Sengupta et al., (2010), Chandrasekhar et al., (2014) produced transgenic plants resistant to sap-sucking insects viz., brown planthopper (BPH), green leafhopper (GLH) and whitebacked planthopper (WBPH) by transferring Allium sativum leaf lectin gene (asal) from garlic, coding for mannose binding homodimeric protein (ASAL) through Agrobacteriummediated genetic transformation of embryogenic calli. Weng et al., (2014) used male sterile lines as transformation materials and the modified/optimized Cry $2 \mathrm{Aa}$ gene along with Bar gene as a selection maker was transferred into 4008S via Agrobacteriummediated method to generate insect-resistant 
and herbicide-tolerant Photoperiod-sensitive genic male sterile rice. Yang et al., (2014) generated transgenic rice "Xiushui 134" that is highly resistant to leaf folder using crylAcl by Agrobacterium mediated transformation method. Jin et al., (2015) developed a highly lepidopteran pest resistant japonica rice variety Jijing 88 by transferring synthetic cry $2 A$ gene via Agrobacterium-mediated transformation. Recently, Manikandan et al., (2016) successfully engineered rice plants expressing synthetic cry $2 A X I$ gene exhibits resistance to rice leaf folder. Lee et al., 2016 produced insect resistant rice transgenic plants by using the construct with the insecticide $c r y l A c$ gene for use in practical agriculture. Similarly, Ling et al., (2016) successfully developed marker-free RSVresistant transgenic plants using a twin TDNA system and RNAi technology, a synthetic cry $2 A$ gene were introduced via Agrobacterium mediated cotransformation into an elite indica restorer line Minghui 86 (Oryza sativa L. ssp. indica).

Table.1 Examples of insect-resistant transgenic rice developed over last few years

\begin{tabular}{|c|c|c|c|}
\hline Variety & Method of Transformation & Gene & References \\
\hline IR-64 & Agrobacterium-mediated & ASAL & Sengupta et al., 2010 \\
\hline Tachisugata & Agrobacterium-mediated & $D B 1 / G 95 A-m A L S$ & Yoshimara et al., 2011 \\
\hline Nanjing 45 & Agrobacterium-mediated & $\begin{array}{l}\text { sbk (modified from } \\
C r y l A(c) \text { ) and sck } \\
\text { (modified from } \\
C p T I \text { ) }\end{array}$ & Zhang et al., 2013 \\
\hline IR-64 & Agrobacterium-mediated & ASAL & $\begin{array}{l}\text { Chandrasekhar et } \\
2014\end{array}$ \\
\hline 4008S & Agrobacterium-mediated & Cry $2 A a$ & Weng et al., 2014 \\
\hline Xiushui 134 & Agrobacterium-mediated & crylAcl & Yang et al., 2014 \\
\hline Jijing 88 & Agrobacterium-mediated & $\operatorname{cry} 2 A$ & Jin et al., 2015 \\
\hline ASD16 & Agrobacterium-mediated & $\operatorname{cry} 2 A X I$ & Manikandan et al., 2016 \\
\hline & & crylAc & Lee et al., 2016 \\
\hline Minghui 86 & Agrobacterium mediated & $\operatorname{cry} 2 A$ & Ling et al., 2016 \\
\hline & Agrobacterium mediated & (pta) and (Bt) & Cui et \\
\hline
\end{tabular}

Pyramiding of multiple genes against the same pest or a range of pests has proved to be very effective to induce sustainable resistance against insect pests. In addition a number of genes other than cry genes viz. plant lectins and proteinase inhibitor genes have been exploited for generation of insect resistant transgenic crops. Cui et al., (2016) successfully obtained broad-spectrum and high insect-resistant transgenic rice by transferring bivalent plant expression vector carrying two insect-resistant genes viz., Pinellia ternate agglutinin gene (pta) and
Bacillus thuringiensis gene (Bt) into rice restorer Fuhui 838. Similarly, highly enhanced insect resistance has been obtained by Zhang et al., (2013) by transferring bivalent plasmid of pCDMARUBA-Hyg, containing two insect-resistance genes, $s b k$ (modified from CrylA(c)) and sck (modified from $C p T I$ ) in super japonica rice Nanjing 45 by Agrobacterium-mediated transformation method.

The commercialization of insect resistant transgenic rice would help in increasing the 
rice production globally as well as in meeting the ambition of food security. Commercialization of insect resistant transgenic rice requires proper field trials. In addition, to reduce the development of insect resistance to transgenic crops, there is an urgent need to explore the strategies for delaying resistance such as integrated pest management as well need to explore alternative sources for pest resistance other than cry genes such as plant lectins.

\section{References}

Alam, M. F., Datta, K., Abrigo, E., Oliva, N., Tu, J, Virmani S S, Datta S.K. 1999. Transgenic insect resistant maintainer line (IR68899B) for improvement of hybrid rice. Plant Cell Rep., 18:572-575.

Alam, M.F., Datta, K., Abrigo, E., Vasquex, A., Senadhira, D., Datta, S.K. 1998. Production of transgenic deepwater indica rice plants expressing a synthetic Bacillus thuringiensis cryIA $(b)$ gene with enhanced resistance to yellow stem borer. Plant Science., 135: 25-30.

Bajaj, S. and Mohanty, A. 2005. Recent advances in rice biotechnology towards genetically superior transgenic rice. Plant Biotechnol. J., 3:275-307

Bharathi, Y., Vijaya, K.S., Pasalu, I.C., Balachandran, S.M., Reddy, V.D. et al., 2011. Pyramided rice lines harbouring Allium sativum (asal) and Galanthus nivalis (gna) lectin genes impart enhanced resistance against major sap-sucking pests. J Biotechnol.,152:6371

Chandrasekhar, K., Vijayalakshmi, M., Vani, K., Kaul, T. and Reddy, M.K. 2014. Biotechnology Letters., 36(5):1059-67. doi: 10.1007/s10529-014-1459-8.

Chen, H., Lin, Y., and Zhang, Q. 2009. Review and prospect of transgenic rice research. Chin. Sci. Bull., 54, 4049-4068. doi: 10.1007/s11434-009-0645-x

Chen, H., Tang, W., Xu, C.G., Li, X.H., Lin, Y.J., Zhang, Q.F. 2005. Transgenic indica rice plants harboring a synthetic Cry $2 A^{*}$ gene of Bacillus thuringiensis exhibit enhanced resistance against lepidopteran rice pests. Theoretical and Applied Genetics., 111: 1330-1337.

Cheng, X., Sardana, R., Kaplan, H., Altosaar, I. 1998. Agrobacterium transformed rice plants expressing synthetic $c r y I A b$ and cryIAc genes are highly toxic to striped stem borer and yellow stem borer. Proc. Natl. Acad. Sci., USA. 95: 2767-2772.

Cui, Y.Q., Li, S.W., Zhang L., Hong, F.; Li Y.F., Zha, R.M. 2016. Breeding and preliminary identification of binary insect-resistant transgenic rice. Journal of Southern Agriculture., 47 (2):169-173.

Datta, K., Vasquez, A., Tu, J., Torrizo, L., Alam, M.F., Olivia, N., Abrigo, E., Khush, G.S., Datta, S.K. 1998. Constitutive and tissue-specific differential expression of $c r y l A b$ gene in transgenic rice plants conferring resistance to rice insect pest. Theor. Appl. Genet., 97: 20-30

Dossa, G.S., Oliva, R., Maiss, E., Vera-Cruz, C., Wydra, K. 2016. High temperature enhances the resistance of cultivated African rice, Oryza glaberrima, to bacterial blight. Plant Dis., 100: 380-387.

FAO. 2009. Production Year Book for 2011. UN. Rome, Italy p: 118.

Fujimoto, H., Itoh, K., Yamamoto, M., Kyozuka, J., Shimamoto, K. 1993. Insect resistant rice generated by introduction of a modified $\delta$-endotoxim gene of Bacillus thuringiensis. Bio/Technology., 11: 11511155

Ghareyazie, B., Alinia, F., Menguito, C.A., Rubia, L.G., de Palma, J.M., Liwanag, E.A., Cohen, M.B., Khush, G.S., Bennett, J. 1997. Enhanced Resistance to Two Stem Borers in Aromatic Rice Containing a Synthetic crylAb Gene. Mol. Breed., 3: 401- 414.

Giri, C. and Vijaya Laxmi, G. 2000. Production of transgenic rice with agronomically useful genes: an assessment. Biotech $A d v ., 18:$ 653-683. 
International Rice Genome Sequencing Project. 2005. The map-based sequence of the rice genome. Nature., 436: 793-800.

Jiang, Y., Sun, L., Jiang, M., Li, K., Song, Y. and Zhu, C. 2013. Production of markerfree and RSV-resistant transgenic rice using a twin T-DNA system and RNAi. $J$. Biosci., 38 573-581] DOI 10.1007/s 12038-013-9349-0

Jin, Y.M., Rui, M.A., Zhi-Jing Y.U., Ling, W et al., 2015. Development of lepidopteran pest-resistant transgenic japonica rice harboring a synthetic cry $2 \mathrm{~A}^{*}$ gene. Journal of Integrative Agriculture., 14. 10.1016/S2095-3119(14)60897-2.

Kathuria, H., Giri, J., Tyagi, H., and Tyagi, A. K. 2007. Advances in Transgenic Rice Biotechnology. Critical Reviews in Plant Science., $26 . \quad 65-103$. $10.1080 / 07352680701252809$.

Khush, G.S. 2003. Productivity improvement in rice. Nutritional Review., 61: 114- 116.

Klausner, A. 1984. Microbial insect control: using bugs to kill bugs. Nat Biotechnol., 2: 408-419

Lee, D.K., Park, S.H., Seong, S.Y. et al., 2016. Plant Biotechnol Rep., 10:391. https://doi.org/10.1007/s11816-016-0410$\mathrm{y}$

Ling, F., Zhou, F., Chen, H., Lin, Y. 2016. Development of Marker-Free InsectResistant Indica Rice by Agrobacterium tumefaciens-Mediated Co-transformation. Frontiers in Plant Science., 7:1608. DOI=10.3389/fpls. 2016.01608

MacLean, J.L., Dawe, D.C., Hardy, B., Hettel, G.P. 2002. Rice Almanac. International Rice Research Institute, Metro Manila., $13-14$.

Manikandan, R., Balakrishnan, N., Sudhakar, D., Udayasuriya, V. 2016. Transgenic rice plants expressing synthetic cry $2 A X 1$ gene exhibits resistance to rice leaffolder (Cnaphalocrosis medinalis). Biotech., 6(1): 10. doi: 10.1007/s13205-015-0315 4

Maqbool, S.B., Husnain, T., Raizuddin, S., Christou, P. 1998. Effective control of yellow rice stem borer and rice leaf folder in transgenic rice indica varieties Basmati 370 and $\mathrm{M} 7$ using novel delta-endotoxin cry2A Bacillus thuringiensis gene. Mol Breed., 4: 501-507.

McPherson, S.A., Perlak, F.J., Fuchs, R.L., Marrone, P.G., Lavrik, P.B., Fischhoff, D.A. 1988. Characterization of the coleopteran specific protein gene of Bacillus thuringiensis var. tenebrionis. Biotechnology., 6: 61-66.

Mukhtar and Hasnain. 2017. Transgenic basmati rice transformed with the Xa21 gene shows resistance against bacterial leaf blight. Turkish Journal of Botany., 41: $1-10$

Nayak, P., Basu, D., Das, S., Basu, A., Ghosh, D., Rascmakrishnan, N.A., Ghosh, M., Sen, S.K. 1997. Transgenic elite indica rice plants expressing cryIAc borer (Scirpophaga incertulas). Proc. Natl. Acad. Sci. USA. 94: 2111-2116.

Sengupta, S., Chakraborti, D., Mondal, H.A., Das, S. 2010. Plant Cell Rep., 29(3):26171. doi: 10.1007/s00299-010-0819-7.

Shu, Q., Ye, G., Cui, H. et al., 2000. Transgenic rice plants with a synthetic crylAb gene from Bacillus thuringiensis were highly resistant to eight lepidopteran rice pest species Molecular Breeding., 6: 433. https://doi.org/10.1023/A:100965802411 4

Shu, Q.Y., Cui, H.R., Ye, G.Y., Wu, D.X., Xia, Y.W., Gao, M.W., Altosaar, I. 2002. Agronomic and morphological characterization of Agrobacterium transformed Bt rice plants. Euphytica., 127: 345-352.

Song, W.Y., Wang, G.L., Chen, L.L., Kim, H.S., Pi, L.Y., Holsten, T., Wang, B., Zhai, W.X., Zhu, H., Fauquet, C. et al., 1995. A receptor kinaselike protein encoded by the rice disease resistance gene, Xa21. Science., 270: 1804-1806.

Tang, W., Chen, H., Xu, C.G., Li, X.H., Lin, Y.J., Zhang, Q.F. 2006. Development of insect-resistant transgenic indica rice with a synthetic CrylC* ${ }^{*}$ gene. Molecular Breeding., 18: 1-10. 
Tu, J., Ona, I., Zhang, Q., Mew, T.W., Khush, G.S., Datta, S.K. 1998. Transgenic rice variety 'IR72' with Xa21 is resistant to bacterial blight. Theor Appl Genet., 97: 31-36.

Tyagi, A.K., Mohanty, A., Bajaj, S., Chaudhury, A., Maheshwari, S.C. 1999. Transgenic rice: A valuable monocot system for crop improvement and gene research. Crit. Rev. Biotechnol., 19: 4179.

Wani, S.H. and Sah, S.K.. 2014. Biotechnology and Abiotic Stress Tolerance in Rice. J Rice Res., 2: e105.

Weng, L., Deng, L., Lia, F., Xiao, G. 2014. Optimization of the Cry2Aa gene and development of insect-resistant and herbicide-tolerant photoperiod-sensitive genic male sterile rice. Czech J. Genet. Plant Breed., 50: 19-25.

Wunn, J., Kloti, A., Burkhardt, P.K., Biswas, G.C., Launis, K., Iglesias, V.A., Potrykus, I. 1996. Transgenic Indica rice breeding line IR58 expressing a synthetic $c r y I A b$ gene from Bacillus thuringiensis provides effective insect pest control. Bio/Technology., 14: 171-176.

Yamamoto, T. and McLaughlin, R.E. 1981. Isolation of a protein from the parasporal crystal of Bacillus thuringiensis var. kurstaki toxic to the mosquito larva Aedes taeniorhynchus. Biochem Biophys Res Commun., 103: 414-421.

Yang, R.F., Bai, J.J., Piao, Z.Z., and Lee, G.S. 2014. Development of Insect-resistant Transgenic Rice with $c r y l A c l$ Gene. Bt Research., 5(4): $1-9$ (doi: 10.5376/bt.2014.05.0002)

Yarasi, B., Sadumpati, V., Immanni, C.P., Reddy, V.D., Rao, K.V. 2008. Transgenic rice expressing Allium sativum leaf agglutinin (ASAL) exhibits high-level resistance against major sap-sucking pests. BMC Plant Biol., 8:102.

Ye, R., Huang, H., Yang, Z., Chen, T., Liu, L., Li, X., Chen, H., Lin, Y. 2009. Development of insect-resistant transgenic rice with CrylC free endosperm. Pest Manag Sci., 65:10151020.

Yoshimura, S., Komatsu, M., Kaku, K., Hori, M., Ogawa, T., Muramoto, K., Kazama, T., Ito, Y., Toriyama, K. 2012. Production of transgenic rice plants expressing Dioscorea batatas tuber lectin 1 to confer resistance against brown planthopper. Plant Biotechnol, 29: 501-504

Zhang, Q.J. Li, C., Liu, S.K., Lai, D., Qi, Q.M., Lu, C.G. 2013. Breeding and Identification of Insect-Resistant Rice by Transferring Two Insecticidal Genes-sbk and sck. Rice Science, 20 (1): 19-24

\section{How to cite this article:}

Nayan Tara, Meena and Manjjri Singal. 2018. Recent Advances in Transgenic Rice Research Technology. Int.J.Curr.Microbiol.App.Sci. 7(03): 3552-3557.

doi: https://doi.org/10.20546/ijcmas.2018.703.408 